\title{
Extubação paliativa: reflexões bioéticas sobre cuidados em fim de vida
}

Taiane do Socorro Silva Natividade ${ }^{1,4}$, Paula Yasmin Camilo Coelho ${ }^{2}$, Danilo Rocha de Aguiar ${ }^{2}$, Gabriela Ladeia da Silva ${ }^{2}$, Rodrigo Batista da Silva ${ }^{3}$, Ana Cristina Vidigal Soeiro ${ }^{4}$

1. Universidade Federal do Pará, Belém/PA, Brasil. 2. Centro Universitário Metropolitano da Amazônia, Belém/PA, Brasil.

3. Fundação Hospital de Clínicas Gaspar Vianna, Belém/PA, Brasil. 4. Universidade Estadual do Pará, Belém/PA, Brasil.

\section{Resumo}

Cuidados paliativos integram um conjunto de abordagens que objetivam incrementar a qualidade de vida diante de uma doença incurável e potencialmente ameaçadora para a vida. Nesse cenário, dentre as terapêuticas utilizadas no cuidado a pacientes críticos, a extubação paliativa é implementada quando as tentativas de desmame da ventilação mecânica falharam, a fim de evitar o prolongamento da vida a qualquer custo. Mesmo com o limitado número de pesquisas sobre o assunto, importantes debates têm emergido no campo biomédico, ético, religioso e legal, trazendo novas reflexões sobre o tema. No Brasil, ainda há muitos entraves para o procedimento, o que inspira o debate bioético.

Palavras-chave: Cuidados paliativos. Ventilação mecânica. Autonomia.

\section{Resumen}

\section{Extubación paliativa: reflexiones bioéticas sobre los cuidados al final de la vida}

Los cuidados paliativos integran un conjunto de enfoques dirigidos a aumentar la calidad de vida ante una enfermedad incurable y potencialmente amenazadora para la vida. En este escenario, entre las terapéuticas utilizadas en el cuidado a pacientes críticos, la extubación paliativa se implementa cuando los intentos de destete de la ventilación mecánica fallan, con el objetivo de evitar prolongar la vida a toda costa. Incluso con el limitado número de investigaciones sobre el asunto, han surgido debates importantes en los campos biomédico, ético, religioso y legal, aportando nuevas reflexiones sobre el tema. En Brasil, aún hay muchos obstáculos frente a este procedimiento, lo que inspira el debate bioético.

Palabras clave: Cuidados paliativos. Ventilación mecánica. Autonomía.

\section{Abstract}

\section{Palliative extubation: bioethical reflections on end-of-life care}

Palliative care is part of a set of approaches aimed at increasing the quality of life, in the face of an incurable and potentially life-threatening disease. In this scenario, among the therapies for critically ill patients, palliative extubation is implemented when all possibilities of weaning from mechanical ventilation have failed, as an alternative to avoid prolonging life at any cost. Despite the limited number of publication on the subject, important debates have emerged in the biomedical, ethical, religious and legal fields, bringing new reflections on the theme. In Brazil, the procedure still faces many obstacles, which make the subject an inspiring field for the bioethical debate.

Keywords: Palliative care. Respiration, artificial. Personal autonomy. 
A progressiva modernização da medicina prolongou a expectativa de vida e diminuiu significativamente a mortalidade ${ }^{1}$. Entretanto, o aumento da longevidade elevou a prevalência de doenças crônicas e degenerativas, refletindo na demanda por cuidados em saúde de forma contínua e integrada. Assim, mesmo diante dos vários triunfos humanos sobre a doença e a morte, viver e morrer com dignidade passaram a ser fonte de importantes reflexões.

$\mathrm{Na}$ atualidade, cuidados paliativos (CP) motivaram importantes debates sobre o uso do conhecimento e da tecnologia biomédica, pois os avanços tecnológicos que salvam vidas também podem mantê-las à custa do sofrimento do paciente. $\mathrm{E}$ isso não ocorre somente porque o tratamento curativo não se mostra possível, mas pelo investimento em procedimentos que pouco contribuem para a melhora da qualidade de vida ${ }^{2}$. Nesse cenário, a obstinação pelo prolongamento da vida suscita questões éticas desafiadoras - não só para os profissionais da medicina, mas para toda a equipe de saúde - quanto às possibilidades e limites de intervenções terapêuticas, um debate que vem ganhando maior visibilidade no campo das discussões bioéticas.

\section{Método}

Trata-se de estudo bibliográfico realizado por meio de revisão narrativa com objetivo de discutir a extubação paliativa (EP) no âmbito dos cuidados em saúde, tendo como eixo de discussão as contribuições da bioética. A pesquisa, que ocorreu entre outubro de 2019 e fevereiro de 2020, buscou trabalhos a partir dos termos "cuidados paliativos", "extubação paliativa", "terminalidade da vida" e "bioética", e considerou artigos publicados em português, inglês e espanhol disponíveis nas bases de dados SciELO, BVS, MedLine e PubMed. Foram selecionados 40 artigos publicados em periódicos, quatros manuais sobre o tema, três resoluções do Conselho Federal de Medicina (CFM), dois capítulos de livro e o atual Código de Ética Médica.

\section{Resultados e discussão}

A consolidação dos CP tem exercido grande influência para o fortalecimento de novas propostas terapêuticas na abordagem de pacientes com doenças incuráveis. Como resultado, alguns procedimentos usados como tentativa de retardar prognósticos inalteráveis passaram a ser questionados, por suas consequências não só ao paciente, mas também à família deste ${ }^{3}$, pois constituem uma unidade de cuidado.

\section{Cuidados paliativos no cenário atual da medicina}

\section{Busca da dignidade do viver e do morrer}

Na ética médica, o conceito "paliativo" (do latim palliare), com sentido de aliviar provisoriamente, adiar ou remediar ${ }^{4}$, relaciona-se à prudência e ao discernimento necessários na escolha das terapêuticas disponíveis. Nas últimas versões do Código de Ética Médica ${ }^{5}$, os CP integraram algumas das diretrizes deontológicas da profissão, delineando o cenário de direitos e deveres de profissionais da medicina no atendimento a pacientes e suas famílias.

No que concerne aos investimentos terapêuticos, recentes versões do Código de Ética Médica resguardam ao profissional o direito de limitar ou suspender procedimentos de prolongamento de vida, caso seja da vontade da pessoa ou de seu representante legal, mediante a realização plena dos CP. As orientações éticas da profissão convergem para a necessidade de possibilitar a realização de CP como modalidade de intervenção transversal às várias especialidades médicas, implicando, sobretudo, o reconhecimento dos limites das intervenções ${ }^{5}$.

Os CP são definidos pela Organização Mundial da Saúde (OMS) como cuidados ativos e integrais prestados a pacientes que não mais respondem ao tratamento curativo. Nessa situação o manejo da dor e dos sintomas é prioritário, como também de questões de ordem psicológica, social e espiritual, tendo como objetivo melhorar a qualidade de vida. Nessa ótica, as medidas terapêuticas são resultado de trabalho em equipe e devem sobretudo controlar os sintomas e proporcionar maior conforto para o paciente, o que implica não apressar, nem tampouco adiar a morte ${ }^{6}$.

Vale dizer que, embora os CP tenham se difundido com mais rapidez no atendimento a pacientes oncológicos, na atualidade se estendem a uma variedade de situações provocadas por mudanças no perfil epidemiológico e demográfico populacional, o que pressupõe sua introdução precoce na linha de cuidados. Entretanto, estudos 
apontam lacunas na formação dos profissionais de saúde diante dos desafios técnicos e pessoais trazidos por essa nova filosofia no campo dos cuidados em fim de vida ${ }^{7-9}$.

\section{Suporte ventilatório nos cuidados em fim de vida}

\section{Delicado equilíbrio entre beneficência e não maleficência}

Os cuidados em fim de vida incluem diferentes abordagens terapêuticas, daí a importância do trabalho em equipe na atenção integral ao paciente. Dependendo das características de cada caso, as intervenções visam à minimização de sintomas, tais como dor, náusea e vômitos, constipação, obstrução intestinal, caquexia, depressão, ansiedade, delirium, agitação terminal, dispneia e hipersecreção respiratória. De modo geral, enquanto algumas ações são recomendadas, outras são evitadas, num delicado equilíbrio entre beneficência e não maleficência, na busca por melhorar a qualidade de vida e a sobrevida de pacientes ${ }^{10,11}$.

Quando todas as tentativas de desmame falharam, prioritariamente nos casos em que há irreversibilidade da condição clínica, a manutenção da ventilação mecânica invasiva apenas adia um desfecho inevitável. Por essa razão, mais recentemente, a retirada da ventilação mecânica tem sido considerada medida terapêutica cujo objetivo é evitar o sofrimento do paciente, resultado principalmente da dor e desconforto associados ao procedimento. Apesar disso, a opção de retirada desse suporte ainda encontra forte resistência no contexto brasileiro, possivelmente em função de seu duplo efeito ${ }^{12,13}$.

A EP, também denominada de extubação compassiva (EC) ${ }^{14,15}$, se aplica aos casos em que é necessário limitar o investimento terapêutico, particularmente quando todas as possibilidades de desmame ventilatório falharam ou o paciente tem prognóstico desfavorável e se encontra em estado grave e irreversível, diante da irresponsividade ao tratamento ${ }^{16}$. Em tais situações, prolongar a vida a qualquer custo seria uma decisão injustificada e, em grande medida, resultado da obstinação terapêutica dos profissionais ${ }^{17}$. Entretanto, apesar dos benefícios envolvidos, achados na literatura indicam que a EC ainda é pouco realizada no Brasil ${ }^{13}$.
Nas unidades de terapia intensiva (UTI) adulta, alguns critérios são considerados na limitação do suporte avançado de vida, incluindo idade avançada, possibilidade de maior comprometimento da qualidade de vida após o procedimento e pontuação em escalas amplamente utilizadas e recomendadas cientificamente - baixa pontuação no escore Karnofsky Performance Status e alta pontuação no escore Simplified Acute Physiology State $3^{18}$. Nesses casos é indicado não iniciar a ventilação mecânica ou retirá-la, visto que o objetivo desse procedimento é prolongar a vida ${ }^{19}$, meta que nem sempre é eticamente aceitável, considerando as repercussões na qualidade de vida do paciente ${ }^{20}$.

Coradazzi e colaboradores ${ }^{3}$ ressaltam a relevância da preparação do procedimento, que deve contemplar três etapas. A primeira consiste em reunião multiprofissional para investigar se existe consenso sobre a necessidade da EP. Nesse caso, a indicação deve se basear nos parâmetros ventilatórios utilizados e na impossibilidade de manutenção da vida após o desmame, mesmo levando em consideração que possivelmente o óbito não será imediato.

$\mathrm{Na}$ segunda etapa a equipe multiprofissional e o paciente e/ou a família deverão se posicionar em relação às intervenções propostas. Nesse momento, que pode demandar mais de um encontro, serão esclarecidas as opções disponíveis, incluindo quando e como será realizada a EP, e, em razão da intensa mobilização emocional, é recomendável que se transmitam as informações à família de modo compreensível, para que eventuais dúvidas sejam sanadas, visando a tomar a decisão de forma compartilhada. Como se trata de uma intervenção com consequências na evolução clínica, é importante incluir familiares nas decisões, particularmente se a participação do paciente nesse processo não for possível ${ }^{21}$.

Na terceira etapa, que compreende a preparação, toda a equipe deve estar mobilizada para a EP, pois será preciso aferir o nível de consciência do paciente no momento da extubação, que pode ser consciente e tranquilo, dormindo ou profundamente sedado. A discussão de casos pela equipe multiprofissional permite planejar conjuntamente as ações, incluindo encontros com a família para revisar diagnóstico e prognóstico e explicar opções de cuidados possíveis, estabelecendo diferenciação entre EP e eutanásia $^{22,23}$. Comunicar-se harmoniosamente aumenta a proximidade com a família e eleva a taxa de sucesso em até $96 \%$, pois, quando a comunicação é eficiente, 
cada profissional pode se posicionar de forma satisfatória durante o procedimento ${ }^{3}$.

Antes da realização da EP, também é importante monitorar o nível de consciência, cessar as medicações de caráter curativo e manter somente os medicamentos necessários ao alívio de dor ou dispneia, além de suspender procedimentos que não alterarão o prognóstico, tais como diálise $^{22}$. Vale destacar que a suspensão da ventilação mecânica não tem a intenção de acelerar a morte do paciente, motivo pelo qual não deve ser confundida com eutanásia. Ao contrário, trata-se de suporte diante da impossibilidade de reversão do quadro clínico, priorizando os cuidados necessários para que o paciente não agonize durante sua morte, sem incorrer em ações cujo malefício supera os benefícios a serem alcançados ${ }^{24,25}$.

No âmbito hospitalar, a EC tem demonstrado impactos positivos a pacientes, famílias e equipes, desde que siga as recomendações vigentes, haja vista que se trata de procedimento complexo ${ }^{3}$, motivo pelo qual deve ser realizada por profissional intensivista ${ }^{12}$. Quando utilizada de forma incorreta, a EP pode levar à dor e dispneia, reações que se pretende evitar quando o procedimento é realizado.

Vale ressaltar que os métodos de retirada diferem na literatura, pois há diferentes medidas de realização da EP. No primeiro caso, recomenda-se a descontinuação imediata, entretanto a literatura aponta maior associação dessa ação a complicações como dor e estresse, o que foge completamente do objetivo primário dos CP. Outra possibilidade é a extubação terminal, realizada de forma a diminuir progressivamente os parâmetros ventilatórios até a extubação completa, sempre com a preocupação de evitar qualquer sinal de desconforto durante ou depois da extubação, o que pode ser alcançado com uso de opioides e/ou benzodiazepínicos e a oxigenoterapia, mantendo-se a sedação adequada ${ }^{3,26,27}$.

No campo da bioética, as preocupações em evitar a distanásia têm impactado em decisões terapêuticas sobre os limites das intervenções, incluindo a EP, de modo a salvaguardar o bem-estar de pacientes. A finitude da vida e as preocupações com a morte e o morrer ganharam maior visibilidade, não mais como componentes da vida biológica, mas como experiências de caráter psicológico, social e espiritual que precisam ser incorporadas como objetivo do cuidado em saúde. Entretanto, mesmo com o avanço dos $\mathrm{CP}$ nas últimas décadas, o Brasil ainda ocupava até recentemente posição modesta de $42^{\circ}$ lugar no ranking de qualidade de morte ${ }^{28}$.

\section{Extubação paliativa e processo de tomada de decisão}

\section{Uma decisão compartilhada?}

No que concerne à relação terapêutica, especialmente em países desenvolvidos, os CP implicaram novas modalidades de relacionamento, com a inclusão de pacientes e familiares no processo de tomada de decisões que anteriormente eram de domínio médico. Essa foi uma das mudanças determinantes para a qualidade do serviço prestado em $\mathrm{CP}^{29}$, sobre o qual, considerando a importância da preservação da autonomia como princípio ético, o CFM aprovou a Resolução $n^{\circ} 1.995 / 2012$, que trata das diretrizes antecipadas de vontade (DAV). Estas definem previamente o desejo do paciente, acarretando o direito de recusar tratamentos que considere agressivos, ainda que esteja incapacitado de expressar livremente e de forma autônoma sua vontade.

Assim, mesmo em situações em que o paciente esteja impossibilitado de se comunicar, seu desejo manifesto deverá ser exposto por seu representante legal para consideração da equipe de saúde ${ }^{30}$. Isso evidencia como a progressiva valorização da autonomia tem impactado significativamente em condutas clínicas e na relação da equipe de cuidados com pacientes e familiares, que passaram a ser incluídos cada vez mais na tomada de decisões ${ }^{31}$. Nesse aspecto, o investimento na melhoria da comunicação é não somente uma estratégia de humanização, mas também reflexo do compromisso e ética profissional na relação com pacientes e suas famílias ${ }^{31,32}$.

De modo geral, a realização da EC também deve ser discutida pela equipe, com a participação do paciente e sua família. Entretanto, a fim de colocar em prática esse ideal, é necessário investigar antecipadamente o desejo do paciente, o que não é comum, especialmente no Brasil ${ }^{33}$. Limitações de ordem moral e legal, além da predominância de um modelo paternalista de relação, ainda fomentam grandes entraves para uma deliberação compartilhada. Assim, decisões são tomadas pela equipe e pela família a depender de suas crenças, expectativas e valores, o que interfere diretamente na escolha por realizar ou não o procedimento ${ }^{19,20}$. 
Cabe ressaltar que o Brasil ainda não dispõe de legislação específica sobre a ortotanásia, nem sobre as Diretivas Antecipadas de Vontade. Embora o CFM tenha aprovado a Resolução $n^{\circ} 1.995 / 2012^{30}$, que trata do tema, ainda não há leis nacionais sobre o assunto, como em outros países, inclusive latino-americanos. Por essa razão, em caso de indicação da EP, é necessário que o paciente passe por uma criteriosa avaliação realizada pelo médico da UTI e por médico independente, procedimento recomendável para a confirmação do prognóstico ${ }^{3,12}$.

Além da avaliação clínica, cabe à equipe multiprofissional prover informações sobre a EP aos acompanhantes e familiares, incluindo o método utilizado e os riscos e benefícios. A falta de esclarecimento pode gerar relutância para a aceitação do procedimento, provocando ansiedade e depressão, assim como outras complicações associadas ao luto $^{21,34}$. Além disso, o diálogo empobrecido com a equipe pode fazer com que familiares e outras pessoas próximas ao paciente se sintam culpados pela morte do ente querido, o que justifica a importância de um diálogo sincero e acolhedor com a equipe ${ }^{35,36}$.

Como o tempo de sobrevivência é variável, a presença e o apoio da família se tornam essenciais e a equipe deve possibilitar a realização dos rituais sociais e de despedida, bem como desejos de fim de vida ${ }^{37}$. Nesse momento, é preciso respeitar a privacidade da família, sendo possível, no caso de pacientes estáveis, a transferência para leitos fora da UTI ${ }^{16,38}$. O contato da equipe de saúde com a família após a morte do paciente é recomendado a fim de melhorar a qualidade do programa no hospital ${ }^{3} \mathrm{e}$ atender a um dos princípios estruturantes dos $\mathrm{CP}$, que prevê o acompanhamento durante o processo de luto ${ }^{10,11}$.

O acompanhamento psicológico da equipe pode ser de grande valia, tendo em vista que os profissionais apresentam valores e expectativas que podem influenciar sua saúde psicológica durante o procedimento devido a reações de contratransferência e em função de questões éticas e religiosas, que também podem interferir em seu julgamento ${ }^{39}$.

\section{Extubação paliativa no contexto das reflexões bioéticas}

Significativa parcela das reflexões bioéticas sobre a finitude humana abrange questionamentos quanto aos limites do prolongamento da vida por meio de intervenções médicas ${ }^{40}$. Tais reflexões têm se intensificado a partir de conceitos atualmente bastante debatidos no campo da saúde, tais como eutanásia ativa, eutanásia passiva, distanásia, ortotanásia e suicídio assistido e sua relação com $\mathrm{CP}$, especialmente por estarem intimamente relacionados à terminalidade da vida ${ }^{41}$.

A expressão "eutanásia" provém do grego e está historicamente associada ao conceito de boa morte, sendo definida como processo pelo qual se abrevia a vida de forma a evitar sofrimento físico ou psicológico. Bélgica, Países Baixos e Suíça já legalizaram sua realização, a qual tornou possível a realização do desejo da morte de um indivíduo, ainda que mediante uma série de condições ${ }^{40}$. A ortotanásia, por sua vez, prevê a interrupção de práticas que visam a prolongar a vida artificialmente por meio de procedimentos que podem causar sofrimento desnecessário ao paciente ${ }^{41}$.

É importante ressaltar que muitas medidas que retardam a morte também podem ser paliativistas, na medida em que são introduzidas a fim de proporcionar maior conforto e dignidade ao paciente. Assim, mesmo considerando o curso natural de uma doença, alguns tratamentos são ofertados como medida terapêutica de controle da dor e de sintomas diversos, sejam eles físicos ou psicológicos ${ }^{42}$. Por outro lado, a distanásia, também denominada "morte difícil", por seu antagonismo à ortotanásia ${ }^{43}$, refere-se ao prolongamento da vida por meios artificiais com a finalidade de retardar o processo de morte natural, causando mais sofrimento ao paciente.

É importante ressaltar que na ortotanásia a doença é o motor causador da morte, diferentemente da eutanásia passiva, na qual a enfermidade não é fatal ou não representa iminente ameaça de morte. Assim, enquanto a eutanásia passiva encurta a vida, a distanásia a prolonga, mesmo quando o paciente não tem mais chances de cura, como resultado da obstinação terapêutica, que reconhecidamente leva à agonia e ao sofrimento ${ }^{17,43}$. Quando considerados os três conceitos, a EC se alinha aos objetivos da ortotanásia, pois não visa a prolongar a vida artificialmente às custas do sofrimento, tampouco alterar o processo natural da morte, haja vista que os tratamentos empreendidos não interferirão mais no curso natural da doença.

Medidas complementares também podem ser associados à EP, a exemplo da sedação paliativa (SP), 
que pode auxiliar na redução dos sintomas respiratórios ${ }^{44,45}$ quando outras formas de cuidado não trazem mais alívio, pois o sofrimento é muitas vezes proveniente da resistência à morte ${ }^{42}$. Outro aspecto a ser considerado refere-se ao desejo de viver ou morrer, que possivelmente também influencia as decisões do paciente, pois este pode optar por manter as medidas de prolongamento de vida, mesmo que não configurem alívio ${ }^{1,42}$. É necessário considerar que, num cenário em que predomina escassez de recursos humanos e materiais para atender à demanda de $\mathrm{CP}$, nem sempre tal opção é viável, o que traz à tona outras questões éticas relevantes.

Vale dizer que as decisões clínicas voltadas à ortotanásia visam prioritariamente a manter o conforto e a dignidade do paciente, bem como o bem-estar da família, evitando situações desconfortáveis e dolorosas pois descontinuar terapias distanásicas não significa interromper o processo de cuidado ${ }^{1}$. Essa diferenciação também implica questões legais, já que a eutanásia é tipificada como crime no Código Penal brasileiro ${ }^{46}$. A dificuldade para implementar a EP no universo de CP também decorre da escassez de equipes qualificadas e em função de lacunas na formação em $\mathrm{CP}^{7-9}$.

Outro fator a ser considerado são as possíveis influências da religião em decisões clínicas da equipe e da família ${ }^{47}$. Considerando que as religiões abordam de forma distinta a terminalidade da vida com base em diferentes perspectivas e juízos de valor, o procedimento pode ser dificultado por crenças e preceitos religiosos que podem afetar a atitude dos familiares, como também as normativas legais do país ${ }^{48}$. Além disso, a realização da EP levanta discussões bioéticas, pois, embora o Código de Ética Médica reconheça o dever médico de respeitar a autonomia do paciente, em algumas situações a decisão final ainda cabe ao profissional, o que aponta muitas contradições.
O CFM aprovou a Resolução $n^{\circ}$ 2.232/2019 ${ }^{49}$, medida controversa para muitos estudiosos por representar um retrocesso no campo dos $\mathrm{CP}$, uma vez que em situações de urgência e emergência, o médico poderá adotar medidas para preservar a vida do paciente, independentemente da recusa terapêutica.

De modo geral, a EP ainda carece de debates que permitam estabelecer parâmetros éticos para sua utilização. Além do conhecimento técnico-científico sobre o tema, deve prevalecer a humanização, o respeito e a compreensão sobre os impactos das ações terapêuticas, em busca de preservar a dignidade humana nos cuidados em fim de vida. A preparação das equipes para atuar em $\mathrm{CP}$, o maior investimento e valorização do processo de consentimento livre e esclarecido e a criação de comitês de bioética clínica despontam como importantes estratégias nessa direção.

\section{Considerações finais}

Embora a EP esteja bem consolidada em países desenvolvidos, no Brasil ainda há poucas pesquisas sobre a temática. Ademais, o debate ético acerca da EP encontra resistência em função de tabus e concepções religiosas, que dificultam sua realização ${ }^{46,47}$. Possivelmente, parte do problema é resultado de questões técnicas, mas também do enlace do tema com valores morais e religiosos envolvidos na temática da terminalidade da vida, mesmo diante dos avanços alcançados com a consolidação dos CP. Que as questões levantadas na presente discussão favoreçam o enfrentamento dos desafios que permeiam a EP, contribuindo com importantes desdobramentos para decisões clínicas no âmbito dos cuidados em fim de vida.

\section{Referências}

1. Kovács MJ. A caminho da morte com dignidade no século XXI. Rev. bioét. (Impr.) [Internet]. 2014 [acesso 5 abr 2021]; 22(1):94-104. DOI: 10.1590/S1983-80422014000100011

2. Burlá C, Py L. Cuidados paliativos: ciência e proteção ao fim da vida. Cad Saúde Pública [Internet]. 2014 [acesso 5 abr 2021];30(6):1-3. DOI: 10.1590/0102-311XPE020614

3. Coradazzi AL, Inhaia CLS, Santana MTEA, Sala AD, Ricardo CP, Suadicani CO et al. Palliative withdrawal ventilation: why, when and how to do it? HPMIJ [Internet]. 2019 [acesso 5 abr 2021];3(1):10-4. DOI: 10.15406/hpmij.2019.03.00141 
4. Houaiss A. Dicionário eletrônico Houaiss da língua portuguesa [CD-ROM]. Versão 2.0. Rio de Janeiro: Objetiva; 2007. Paliativo.

5. Conselho Federal de Medicina. Código de Ética Médica: Resolução CFM n ${ }^{\circ} 2.217$, de 27 de setembro de 2018, modificada pelas Resoluções CFM n 2.222/2018 e 2.226/2019 [Internet]. Brasília: CFM; 2019 [acesso 5 abr 2021]. Disponível: https://portal.cfm.org.br/images/PDF/cem2019.pdf

6. World Health Organization. Palliative care: the solida facts [Internet]. Genebra: 2004 [acesso 9 ago 2021]. Disponível: https://bit.ly/2X6DcWt

7. Dalpai D, Mendes FF, Asmar JAVN, Carvalho PL, Loro FL, Branco A. Pain and palliative care: the knowledge of medical students and the graduation gaps. Rev Dor [Internet]. 2017 [acesso 5 abr 2021];18(4):307-10. DOI: $10.5935 / 1806-0013.20170120$

8. Cruz RAO, Arruda AJCG, Agra G, Costa MML, Nóbrega VKM. Reflexões acerca dos cuidados paliativos no contexto da formação em enfermagem. Rev enferm UFPE on line [Internet]. 2016 [acesso $5 \mathrm{abr}$ 2021];10(8):3101-7. Disponível: https://bit.ly/3yF1rsQ

9. Carroll T, Weisbord N, O'Connor A, Quill T. Primary palliative care education: a pilot survey. Am J Hosp Palliat Care [Internet]. 2018 [acesso 5 abr 2021];35(4):565-9. DOI: 10.1177/1049909117723618

10. Matsumoto DY. Cuidados paliativos: conceito, fundamentos e princípio. In: Academia Nacional de Cuidados Paliativos. Manual de cuidados paliativos. Rio de Janeiro: ANCP; 2009. p. 14-9.

11. Ferreira GD, Mendonça GN. Cuidados paliativos: guia de bolso. São Paulo: ANCP; 2017.

12. Kok VC. Compassionate extubation for a peaceful death in the setting of a community hospital: a case-series study. Clin Interv Aging [Internet]. 2015 [acesso 5 abr 2021];10:679-85. DOI: 10.2147/CIA.S82760

13. Bitencourt AGV, Dantas MP, Neves FBCS, Almeida AM, Melo RMV, Albuquerque LC et al. Condutas de limitação terapêutica em pacientes internados em unidade de terapia intensiva. Rev Bras Ter Intensiva [Internet]. 2007 [acesso 5 abr 2021];19(2):137-43. DOI: 10.1590/S0103-507X2007000200001

14. Temel JS, Greer JA, Muzikansky A, Gallagher ER, Admane S, Jackson VA et al. Early palliative care for patients with metastatic non-small-cell lung cancer. N Engl J Med [Internet]. 2010 [acesso 5 abr 2021];363(8):733-42. DOI: 10.1056/NEJMoa1000678

15. Smith S, Brick A, O'Hara S, Normand C. Evidence on the cost and cost-effectiveness of palliative care: a literature review. Palliat Med [Internet]. 2014 [acesso 5 abr 2021];28(2):130-50. DOI: $10.1177 / 0269216313493466$

16. Coradazzi ALT. Extubação paliativa. In: Coradazzi AL, Santana MTEA, Caponero R, organizadores. Cuidados paliativos: diretrizes para melhores práticas. São Paulo: MG; 2019. p. 193-202.

17. Oliveira SG, Quintana AM, Bertolino KCO. Reflexões acerca da morte: um desafio para a enfermagem. Rev Bras Enferm [Internet]. 2010 [acesso 5 abr 2021];63(6):1077-80. DOI: 10.1590/S003471672010000600033

18. Mazutti SRG, Nascimento AF, Fumis RRL. Limitação de suporte avançado de vida em pacientes admitidos em Unidade de Terapia Intensiva com cuidados paliativos integrados. Rev Bras Ter Intensiva [Internet]. 2016 [acesso 5 abr 2021];28(3):294-300. DOI: 10.5935/0103-507X.20160042

19. Smedira NG, Evans BH, Grais LS, Cohen NH, Lo B, Cooke M et al. Withholding and withdrawal of life support from the critically ill. N Engl J Med [Internet]. 1990 [acesso 5 abr 2021];322(5):309-15. DOI: 10.1056/ NEJM199002013220506

20. Lage JSS, Pincelli ASM, Furlan JAS, Ribeiro DL, Marconato RS. Extubação paliativa em unidade de emergência: relato de caso. Rev. bioét. (Impr.) [Internet]. 2019 [acesso 5 abr 2021];27(2):313-7. DOI: 10.1590/ 1983-80422019272315

21. Hinkle LJ, Bosslet GT, Torke AM. Factors associated with family satisfaction with end-of-life care in the ICU: a systematic review. Chest [Internet]. 2015 [acesso 5 abr 2021];147(1):82-93. DOI: 10.1378/chest.14-1098

22. O'Mahony S, McHugh M, Zallman L, Selwyn P. Ventilator withdrawal: procedures and outcomes. Report of a collaboration between a critical care division and a palliative care service. J Pain Symptom Manage [Internet]. 2013 [acesso 5 abr 2021];26(4):954-61. DOI: 10.1016/S0885-3924(03)00333-6 
23. Campbell ML, Weissman DE, Nelson JE. Palliative care consultation in the ICU \#253. J Palliat Med [Internet]. 2012 [acesso 5 abr 2021];15(6):715-6. DOI: 10.1089/jpm.2012.9582

24. Sanches KMS, Seidl EMF. Ortotanásia: uma decisão frente à terminalidade. Interface Comun Saúde Educ [Internet]. 2013 [acesso 5 abr 2021];17(4):23-34. DOI: 10.1590/S1414-32832013000100003

25. Xavier MS, Miziara CSMG, Miziara ID. Terminalidade da vida: questões éticas e religiosas sobre a ortotanásia. Saúde, Ética \& Justiça [Internet]. 2014 [acesso 5 abr 2021];19(1):26-34. DOI: 10.11606/ issn.2317-2770.v19i1p26-34

26. Robert R, Le Gouge A, Kentish-Barnes N, Cottereau A, Giraudeau B, Adda M et al. Terminal weaning or immediate extubation for withdrawing mechanical ventilation in critically ill patients (the ARREVE observational study). Intensive Care Med [Internet]. 2017 [acesso 5 abr 2021];43(12):1793-807. DOI: 10.1007/s00134-017-4891-0

27. Walling AM, Asch SM, Lorenz KA, Roth CP, Barry T, Kahn KL, Wenger NS. The quality of care provided to hospitalized patients at the end of life. Arch Intern Med [Internet]. 2010 [acesso 5 abr 2021];170(12):1057-63. DOI: 10.1001/archinternmed.2010.175

28. The 2015 quality of death index. Ranking palliative care across the world. The Economist Inteligence Unit [Internet]. 2015 [acesso 10 ago 2021]. Disponível: https://bit.ly/3xvmdK4

29. Costa Filho RC, Costa JLF, Gutierrez FLBR, Mesquita AF. Como implementar cuidados paliativos de qualidade na Unidade de Terapia Intensiva. RBTI (Impr.) [Internet]. 2008 [acesso 5 abr 2021];20(1):88-92. DOI: 10.1590/S0103-507X2008000100014

30. Conselho Federal de Medicina. Resolução CFM n ${ }^{\circ}$ 1.995, de 31 de agosto de 2012 [Internet]. Brasília: CFM; 2012 [acesso 5 abr 2021]. Disponível: https://bit.ly/2U6EIXz

31. Organização Mundial da Saúde. Envelhecimento ativo: uma política de saúde [Internet]. Brasília: Organização Pan-Americana de Saúde; 2005 [acesso 5 abr 2021]. Disponível: https://bit.ly/37xFgc4

32. Costa RS, Santos AGB, Yarud SD, Sena ELS, Boery RNSO. Reflexões bioéticas acerca da promoção de cuidados paliativos a idosos. Saúde Debate [Internet]. 2016 [acesso 5 abr 2021];40(108):170-7. DOI: 10.1590/ 0103-1104-20161080014

33. Hassegawa LCU, Rubira MC, Rubira APA, Katsuragawa TH, Gallo JH, Nunes RML. Abordagens e reflexões sobre diretivas antecipadas da vontade no Brasil. Rev Bras Enferm [Internet]. 2019 [acesso 5 abr 2021];72(1):266-75. DOI: 10.1590/0034-7167-2018-0347

34. Schmidt M, Azoulay E. Having a loved one in the ICU: the forgotten family. Curr Opin Crit Care [Internet]. 2012 [acesso 5 abr 2021];18(5):540-7. DOI: 10.1097/MCC.0b013e328357f141

35. Truog RD, Meyer EC, Burns JP. Towards interventions to improve end-of-life care in the pediatrics intensive care unit. Crit Care Med [Internet]. 2006 [acesso 5 abr 2021];34(supl 11):373-9. DOI: 10.1097/ 01.CCM.0000237043.70264.87

36. Prendergast TJ, Puntillo KA. Withdrawal of life support: intensive caring at the end of life. JAMA [Internet]. 2002 [acesso 5 abr 2021];288(21):2732-40. DOI: 10.1001/jama.288.21.2732

37. Floriani CA. Moderno movimento hospice: kalotanásia e o revivalismo estético da boa morte. Rev. bioét. (Impr.) [Internet]. 2013 [acesso 5 abr 2021];21(3):397-404. Disponível: https://bit.ly/3fRWFAR

38. Coelho CBT, Yankaskas JR. Novos conceitos em cuidados paliativos na unidade de terapia intensiva. Rev Bras Ter Intensiva [Internet]. 2017 [acesso 5 abr 2021];29(2):222-30. DOI: 10.5935/ 0103-507X.20170031

39. Porto G, Lustosa MA. Psicologia hospitalar e cuidados paliativos. Rev SBPH [Internet]. 2010 [acesso 5 abr 2021];13(1):76-93. Disponível: https://bit.ly/3xAJHNS

40. Felix ZC, Costa SFG, Alves AMPM, Andrade CG, Duarte MCS, Brito FM. Eutanásia, distanásia e ortotanásia: revisão interativa da literatura. Ciênc Saúde Colet [Internet]. 2013 [acesso 5 abr 2021];18(9):2733-46. Disponível: https://bit.ly/3AxBPhU

41. Kovács MJ. Bioética nas questões da vida e da morte. Psicol USP [Internet]. 2003 [acesso 5 abr 2021];14(2):115-67. Disponível: https://bit.ly/3yCHPpi 
42. Kovác MJ. Morte com dignidade. In: Fukumitsu KO, organizadora. Vida, morte e luto: atitudes brasileiras. São Paulo: Summus; 2018. p. 29-48.

43. Menezes MB, Selli L, Alves JS. Distanásia: percepção dos profissionais da enfermagem. Rev Latinoam Enferm [Internet]. 2009 [acesso 5 abr 2021];17(4):443-8. DOI: 10.1590/S0104-11692009000400002

44. Morita T, Chinone Y, Ikenaga M, Miyoshi M, Nakaho T, Nishitateno $\mathrm{K}$ et al. Ethical validity of palliative sedation therapy: a multicenter, prospective, observational study conducted on specialized palliative care units in Japan. J Pain Symptom Manage [Internet]. 2005 [acesso 5 abr 2021];30(4):308-19. DOI: 10.1016/ j.jpainsymman.2005.03.016

45. Mazer MA, Alligood CM, Wu Q. The infusion of opioids during terminal withdrawal of mechanical ventilation in the medical intensive care unit. J Pain Symptom Manage [Internet]. 2010 [acesso 5 abr 2021];42(1):44-51. DOI: 10.1016/j.jpainsymman.2010.10.256

46. Brasil. Decreto-Lei $n^{\circ} 2.848$, de 7 de dezembro de 1940. Código Penal. Diário Oficial da União [Internet]. Rio de Janeiro, 1940 [acesso 5 abr 2021]. Disponível: https://bit.ly/2U4z1cE

47. De La Longuiniere ACF, Yarid SD, Silva ECS. Influência da religiosidade/espiritualidade dos profissionais da saúde na valorização da dimensão espiritual do paciente crítico. Rev Enferm UFPE on line [Internet]. 2017 [acesso 5 abr 2021];11(6):2510-7. Disponível: https://bit.ly/3fMMptt

48. Santos DA, Almeida ERP, Silva FF, Andrade LHC, Azevêdo LA, Neves NMBC. Reflexões bioéticas sobre a eutanásia a partir de caso paradigmático. Rev. bioét. (Impr.) [Internet]. 2014 [acesso 5 abr 2021];22(2):367-2. DOI: $10.1590 / 1983-80422014222018$

49. Conselho Federal de Medicina. Resolução CFM n 2.232, de 26 de setembro de 2019 [Internet]. Brasília: CFM; 2019 [acesso 5 abr 2021]. Disponível: https://bit.ly/37y8XtG

Taiane do Socorro Silva Natividade - Graduada - ts.natividade@gmail.com

(D) 0000-0002-9746-7568

Paula Yasmin Camilo Coelho - Graduada - paulaycoelho@gmail.com

(D) 0000-0003-0545-1436

Danilo Rocha de Aguiar - Graduado - daniloaguiarr20@gmail.com

(D) 0000-0002-0699-2122

Gabriela Ladeia da Silva - Graduada - gabiladeia@hotmail.com

(iD) 0000-0003-0801-3335

Rodrigo Batista da Silva - Graduado - rodrigo.batista.14@hotmail.com

(D) 0000-0002-1535-9839

Ana Cristina Vidigal Soeiro - Graduada - acsoeiro1@gmail.com

(D) 0000-0002-1669-3839

Correspondência

Taiane do Socorro Silva Natividade - Universidade do Estado do Pará. Travessa Perebebuí, 2623, Marco CEP 66087-662. Belém/PA, Brasil.

Participação dos autores

Taiane do Socorro Silva Natividade idealizou o tema e participou da redação e revisão final do artigo. Paula Yasmin Camilo Coelho, Danilo Rocha de Aguiar, Gabriela Ladeia da Silva e Rodrigo Batista da Silva contribuíram com a redação e a revisão final do artigo. Ana Cristina Vidigal Soeiro orientou o trabalho e participou da redação e revisão final do artigo.

Recebido: 18.3 .2020

Revisado: $\quad 3.8 .2021$

Aprovado: $\quad 4.8 .2021$ 